\title{
Intravenous Epoprostenol for Management of Pulmonary Arterial Hypertension during Pregnancy
}

\author{
Julia Timofeev, MD ${ }^{1}$ George Ruiz, MD² Melissa Fries, MD ${ }^{1}$ Rita W. Driggers, MD
}

${ }^{1}$ Division of Maternal-Fetal Medicine, Department of Obstetrics and Gynecology, MedStar Washington Hospital Center, MedStar Health,

Address for correspondence Julia Timofeev, MD, 110 Irving Street Washington, District of Columbia

2 Department of Cardiology, MedStar Washington Hospital Center, MedStar Health, Washington, District of Columbia

Am J Perinatol Rep 2013;3:71-74.

\begin{abstract}
Keywords

- epoprostenol

- pulmonary hypertension

- prostaglandin

- prostacyclin

Background Pulmonary arterial hypertension carries a high risk of mortality in pregnancy. Recent advances in treatment may improve disease course and allow for successful management of the pregnancy.

Case Report We present the case of a 20-year-old gravida 1, para 0 with diagnosis of severe primary pulmonary hypertension. The patient was managed with epoprostenol (prostacyclin) infusion via an indwelling catheter, which was initiated at 23 weeks' gestation. The dose was adjusted to the patient's symptoms and a successful vaginal delivery was achieved at 36 weeks' gestation. Although maternal postpartum course was uncomplicated, unexplained neonatal demise occurred at 11 days of life.

Conclusion Successful management of pulmonary hypertension in pregnancy can be accomplished with a multidisciplinary approach and intensive therapy. Long-term effects of epoprostenol on fetal or neonatal well-being are unknown.
\end{abstract}

Pulmonary arterial hypertension, defined by pulmonary artery (PA) pressure $>25 \mathrm{~mm} \mathrm{Hg}$ at rest or $>30 \mathrm{~mm} \mathrm{Hg}$ during exercise, predominantly affects women of reproductive age and carries a mortality rate of 30 to $56 \%$ in pregnancy. ${ }^{1}$ The disease is characterized by increased vasoconstriction and poor vasodilation due to significant endothelial dysfunction with resultant remodeling of the walls of the pulmonary arteries. Prior to recent introduction of several classes of medications, pulmonary hypertension carried a dismal survival from the time of diagnosis. Currently, even with advanced therapy, the mortality still remains high, approximately $15 \%$ during the first year after diagnosis in nonpregnant patients.

Women are counseled to avoid pregnancy given the grave prognosis in a setting of compromised pulmonary circulation and significant cardiovascular demands of pregnancy and labor. Adverse neonatal outcomes are usually the result of prematurity due to indicated delivery for either maternal or fetal indications. Although often used in pregnant patients with pulmonary hypertension, there are very little data on effect of prostacyclin on fetal intrauterine environment. We report a case of severe primary pulmonary hypertension managed with intravenous epoprostenol.

\section{Case Report}

A 20 -year-old gravida 1 , para 0 with primary pulmonary hypertension diagnosed at the age of 9 years old was referred to our center at 23 weeks' gestational age due to worsening dyspnea, chest tightness, and syncope. She had been managed with nifedipine preconception and through the early second trimester, at which time she was transitioned to sildenafil due to worsening of her symptoms. Upon presentation to our institution at 23 weeks, echocardiogram showed a dilated right ventricle (RV) with reduced function and increased $\mathrm{RV}$ received

December 21, 2012 accepted after revision

February 2, 2013

published online

March 18, 2013
DOI http://dx.doi.org/

10.1055/s-0033-1338169. ISSN 2157-6998.
Copyright $\odot 2013$ by Thieme Medical Publishers, Inc., 333 Seventh Avenue, New York, NY 10001, USA. Tel: +1(212) 584-4662.
License terms

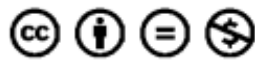


pressure, and ejection fraction (EF) of 55 to $70 \%$. Right heart catheterization confirmed severe pulmonary hypertension, with $50 \%$ reduction in PA pressures with nitric oxide (PA pressures decreased from $70 / 34$ to $36 / 20 \mathrm{~mm} \mathrm{Hg}$, and cardiac output increased from 5.8 to $7.2 \mathrm{~L} / \mathrm{min}$ ). Sildenafil therapy was discontinued at that time as her symptoms were not well controlled, and the patient was placed on epoprostenol intravenous infusion and therapeutic dose subcutaneous heparin (15,000 U twice a day, goal partial thromboplastin time 2 to 2.5 times of normal, goal anti-Xa 0.3 to 0.7 ) due to the hypercoagulable state of pregnancy and decreased blood flow in the pulmonary vessels with worsening pulmonary hypertension. The epoprostenol was titrated up slowly, with fetal monitoring with nonstress test and Doppler evaluation of umbilical and uterine arteries performed during and following each incremental increase in dose.

The patient was managed in an outpatient setting after initiating prostacyclin therapy with evaluation two times a week in the labor and delivery unit for fetal monitoring and with the cardiology department for medication adjustment. A single lumen Hohn catheter, Bard Access Systems, Salt Lake City, UT, was placed in the right internal jugular vein for medication administration, and infusion was performed with CADD ambulatory infusion pump (Smiths Medical, Dublin, $\mathrm{OH}$, USA). Fetal growth was followed closely and the epoprostenol was incrementally increased weekly to a final dose of $25 \mathrm{ng} / \mathrm{kg} / \mathrm{min}$. The estimated fetal weight was in the 32nd percentile at 23 weeks. Fetal weight decreased to the 4th percentile 2 weeks after starting epoprostenol, with normal amniotic fluid index and umbilical and middle cerebral Doppler studies at that time. However, the fetal growth rebounded as prostacyclin was continued, and at 35 weeks, estimated fetal weight was at the 43rd percentile with epoprostenol dose at $25 \mathrm{ng} / \mathrm{kg} / \mathrm{min}$. All antepartum testing and Doppler studies of the fetus were reassuring.

The delivery plan was developed by a multidisciplinary team that included maternal-fetal medicine specialists, cardiologists, anesthesiologists, and neonatologists. Given that the patient was tolerating pregnancy well, induction of labor with invasive cardiac monitoring via Swan-Ganz catheter, continued epoprostenol infusion, nitric oxide immediately available, and an assisted second stage of labor was planned at 36 weeks. PA catheter was placed prior to induction of labor and PA pressure was 27/10 while on epoprostenol. Epidural was carefully titrated with monitoring of PA and systemic blood pressures to avoid a drop in cardiac output. The patient progressed steadily in labor and delivered a viable male neonate with Apgar scores of 7 and 8 at 1 and 5 minutes, respectively, via low forceps-assisted vaginal delivery. Immediately after delivery, patient had a prompt increase in PA pressure to 80/30, which responded to increase in epoprostenol to $30 \mathrm{ng} / \mathrm{kg} / \mathrm{min}$ and administration of furosemide, and subsequent postpartum PA pressures ranged from 30 to 40/10 to 20 . The patient was then transferred to the cardiac care unit for close monitoring in the postpartum period. Echocardiogram after delivery showed an EF of 55 to 60\%, reduced RV function, and RV dilation. The Swan-Ganz catheter was discontinued after 3 days, and the patient was discharged on epoprostenol therapy and warfarin with planned transition back to nifedipine or sildenafil postpartum after repeat right heart catheterization. The patient received Implanon, Merk \& Co., Inc, Whitehouse Station, NJ, for contraception at the time of discharge. The infant was evaluated in the neonatal intensive care unit for 3 days and was discharged home with the mother on the sixth day of life. Unexplained neonatal demise occurred at 11 days of life, which was attributed to sudden infant death syndrome. The autopsy was unremarkable with cardiac evaluation revealing normal cardiac chambers with patent foramen ovale and probe patent ductus arteriosus.

Approximately 8 weeks postpartum, the patient was readmitted for repeat right heart catheterization, which again showed moderate pulmonary hypertension with 33\% reduction in PA pressure and $40 \%$ increase in cardiac output with nitric oxide challenge (PA pressure decreased from 47/20 to $32 / 12 \mathrm{~mm} \mathrm{Hg}$, and cardiac output increased from 6.9 to $10.8 \mathrm{~L} /$ min). Echocardiogram at that time showed EF of 55 to $60 \%$, and RV overload with reduced RV systolic function. The patient was slowly weaned off epoprostenol over 12 hours and restarted on nifedipine $10 \mathrm{mg}$ three times a day, which she tolerated well.

\section{Discussion}

Pulmonary arterial hypertension predominantly affects women of reproductive age and carries a mortality rate as high as $56 \%$ in pregnancy. It is defined by PA pressure $>25$ $\mathrm{mm} \mathrm{Hg}$ at rest or $>30 \mathrm{~mm} \mathrm{Hg}$ during exercise and is classified into five subgroups: idiopathic, familial, or related to connective tissue disease; associated with left heart disease; associated with lung disease (e.g., chronic obstructive pulmonary disease); associated with chronic thrombotic disease; and associated with other miscellaneous conditions (histiocytosis, sarcoidosis, etc.). ${ }^{2}$

The disease is characterized by increased vasoconstriction and poor vasodilation due to remodeling of the walls of the pulmonary arteries. Smooth muscle proliferation decreases the size of vessel lumen with an associated increased resistance to blood flow. Overexpression of endothelin-1 plays an important role in inflammation and vasoconstriction, and a decrease in the production of vasoactive substances (such as prostacyclin and nitric oxide) contributes to the underlying pathophysiology of endothelial dysfunction. Advanced targeted therapies focus on endothelin, nitric oxide, and prostacyclin pathways, resulting in development of endothelinreceptor antagonists, prostacyclin analogues, phosphodiesterase 5 inhibitors, and the use of nitric oxide to mitigate vessel wall damage. Increased thrombotic risk from the primary disease is treated with anticoagulation to prevent thrombosis. ${ }^{3}$ Calcium channel blockers can be used in a small proportion of patients who have vasospasm identified as a contributor to their pulmonary hypertension.

Of the available drugs for treatment of pulmonary arterial hypertension, endothelin-receptor antagonist (bosentan) is contraindicated in pregnancy because of concerns for increased rates of congenital malformations in animal studies. Calcium channel blockers are considered safe for use in pregnancy; however, only about $10 \%$ of patients with 
pulmonary hypertension respond to this type of therapy with a sustained effect. $^{3}$ Several case reports in the literature describe the use of prostacyclins in pregnant patients. ${ }^{4-6}$ The short half-life of these compounds require frequent administration, either by inhalation or continuous intravenous (IV) infusion. Nitric oxide and sildenafil have also been used successfully either as sole agents or as adjuncts to other medications during the intrapartum course.

Prior to the recent introduction of several classes of medications, pulmonary hypertension carried a dismal survival from the time of diagnosis (median 2.8 years in nonpregnant patients). Currently, even with advanced therapy, the mortality remains high, approximately $15 \%$ during the first year after the diagnosis. ${ }^{7}$ In a systematic review by Weiss et al from 1978 to $1996,{ }^{1}$ maternal mortality rate was $36 \%$ in Eisenmenger syndrome, $30 \%$ in primary pulmonary hypertension, and 56\% in women with secondary vascular pulmonary hypertension. Late diagnosis and delayed hospital admission were independent risk factors for maternal mortality for all patients with pulmonary arterial hypertension, regardless of the underlying etiology. After the introduction of epoprostenol in 1996, with a multidisciplinary and aggressive approach to treatment, mortality rates have improved. Bédard and colleagues compared maternal mortality rates for the recent decade (1997 to 2007) to 1978 to 1996 data reported by Weiss et al, ${ }^{1}$ demonstrating the maternal mortality rate of $17 \%$ in primary pulmonary hypertension, $28 \%$ for patients with Eisenmenger syndrome, and 33\% for those with secondary pulmonary hypertension. ${ }^{7}$

Women with pulmonary hypertension are counseled to avoid pregnancy given the grave prognosis in a setting of compromised, high-resistance pulmonary circulation and significant cardiovascular demands of pregnancy and parturition. Normal physiologic changes in pregnancy include a rise in total blood volume and cardiac output by about $50 \%$, with a concomitant decrease in systemic vascular resistance from vasodilation to accommodate the increase in volume. Maternal heart rate is also increased by 10 to 20 beats per minute, contributing to the elevated cardiac output, and rising circulatory blood volume results in increased preload. During labor, cardiac output may increase an additional 30 to $50 \%$, resulting in an overall increase of $80 \%$ over prepregnancy values. Following the delivery of the neonate, autotransfusion of approximately $500 \mathrm{~mL}$ occurs due to return of uteroplacental blood into maternal circulation, and the pressure from the gravid uterus is relieved, resulting in aorto-caval decompression with increased blood flow to the right side of the heart. ${ }^{2}$ These significant hemodynamic shifts are not well tolerated in patients with pulmonary hypertension, and death may occur due to right heart failure, arrhythmias, PA thrombosis, or pulmonary hypertensive crisis. The hypercoagulable state of pregnancy due to increased levels of certain clotting factors, reduced levels of protein $\mathrm{S}$, and resistance to activated protein $\mathrm{C}$ adds to the thrombotic risk from decreased blood flow in the pulmonary vessels due to narrowing and increased resistance with worsening of the pulmonary hypertension. Third trimester, labor and delivery, and especially the postpartum period are the most critical periods, with the majority of maternal deaths reported within the first 10 days after delivery. ${ }^{1}$

The timing of medical therapy as well as agents used in pregnant women with pulmonary hypertension differ across reports. ${ }^{4-7}$ Initiation of treatment prior to acute decompensation is associated with improved outcomes. Our patient had been controlled on calcium channel blockers prior to pregnancy; however, her symptoms progressed and she was started on outpatient sildenafil and then changed to prostacyclin after increasing presyncopal events. Epoprostenol (prostacyclin) or prostaglandin I2 stimulates cyclic adenosine monophosphate, resulting in relaxation of vascular smooth muscle. It is also a potent inhibitor of platelet aggregation, with results lasting 20 to 30 minutes. Due to its short half-life, it must be administered by continuous IV infusion. Outpatient management was possible for our patient with the use of the CADD ambulatory pump and indwelling Hohn catheter. It is by far the most commonly used drug agent for pulmonary hypertension in pregnancy $(62 \%){ }^{7}$ Animal safety data do not show adverse effects on fetal development in doses up to 4.8 times the maximum human dose in rats and rabbits. However, administration of prostacyclin to near-term pregnant sheep resulted in fetal acidemia thought to be due to shunting of blood away from the uterus due to maternal systemic arteriolar dilatation. ${ }^{8}$

Although the mode of delivery for patients with pulmonary hypertension is controversial, vaginal delivery was planned for our patient to avoid excessive bleeding and large fluid volume shifts that occur during cesarean delivery. Regional anesthesia to control pain and to avoid pain-related tachycardia was vital, but extreme care was required during dosing of the epidural to avoid sudden maternal hypotension (and decrease in cardiac output) due to systemic vasodilation. The second stage was also shortened with the use of operative vaginal delivery to prevent hypertension and tachycardia possibly resulting from maternal pushing efforts. A sudden rise in PA pressures immediately after delivery has been reported and was also observed in our patient. She responded well to increased dose of IV epoprostenol and furosemide, and no other additional treatment was required.

Previous case reports involving the use of prostacyclin do not report adverse neonatal outcomes, as most neonatal complications are attributed to prematurity resulting from medically indicated preterm delivery for maternal or fetal indications. Review of literature by Weiss et al and Bédard et al showed neonatal survival rate of nearly $90 \%$ regardless of the subgroup of pulmonary hypertension, with fetal growth restriction reported in 3 to $33 \%$ of pregnancies. ${ }^{1,7}$ In our case, although maternal outcome was favorable, neonatal death occurred shortly after birth, which was attributed to sudden infant death syndrome. Although no specific studies exist on possible lactation effects of epoprostenol, prostacyclin is not active orally and would not be expected to be active if consumed in breast milk.

Successful management of pregnancy in a setting of pulmonary hypertension is possible with close monitoring, targeted medical therapy, and multidisciplinary approach. Long-term fetal and neonatal effects of prolonged 
74 Pulmonary Hypertension in Pregnancy Timofeev et al.

prostacyclin therapy are unknown. Pulmonary hypertension in pregnancy still carries a high risk of mortality and pregnancy should be avoided if possible.

\section{References}

1 Weiss BM, Zemp L, Seifert B, Hess OM. Outcome of pulmonary vascular disease in pregnancy: a systematic overview from 1978 through 1996. J Am Coll Cardiol 1998;31:1650-1657

2 Madden BP. Pulmonary hypertension and pregnancy. Int J Obstet Anesth 2009;18:156-164

3 Humbert M, Sitbon O, Simonneau G. Treatment of pulmonary arterial hypertension. N Engl J Med 2004;351:1425-1436
4 Bendayan D, Hod M, Oron G, et al. Pregnancy outcome in patients with pulmonary arterial hypertension receiving prostacyclin therapy. Obstet Gynecol 2005;106(5 Pt 2):1206-1210

5 Bildirici I, Shumway JB. Intravenous and inhaled epoprostenol for primary pulmonary hypertension during pregnancy and delivery. Obstet Gynecol 2004;103(5 Pt 2):1102-1105

6 Avdalovic M, Sandrock C, Hoso A, Allen R, Albertson TE. Epoprostenol in pregnant patients with secondary pulmonary hypertension: two case reports and a review of the literature. Treat Respir Med 2004;3:29-34

7 Bédard E, Dimopoulos K, Gatzoulis MA. Has there been any progress made on pregnancy outcomes among women with pulmonary arterial hypertension? Eur Heart J 2009;30:256-265

8 Parisi VM, Walsh SW. Fetal vascular responses to prostacyclin. Am J Obstet Gynecol 1989;160:871-876, discussion 876-878 